\title{
Correction to: Clinical and economic effects of an antimicrobial stewardship intervention in a surgical intensive care unit
}

\author{
Hartmut Stocker ${ }^{1,4} \cdot$ Cornelia Mehlhorn $^{2} \cdot$ Kati Jordan $^{2} \cdot$ Leila Eckholt $^{3} \cdot$ Laura Jefferys $^{1} \cdot$ Keikawus Arastéh $^{1}$
}

Published online: 20 May 2020

c) Springer-Verlag GmbH Germany, part of Springer Nature 2020

\section{Correction to: Infection}

https://doi.org/10.1007/s15010-020-01421-8

The original version of this article unfortunately contained a mistake

The spelling of the name Kati Jordan was incorrect.

The original article has been corrected.

\section{Hartmut Stocker}

hartmut.stocker@sjk.de

1 Department of Infectious Diseases, Vivantes AugusteViktoria-Klinikum, Rubensstraße 125, 12157 Berlin,

Germany

2 Department of Anaesthesiology and Intensive Care, Vivantes Auguste-Viktoria-Klinikum, Rubensstraße 125, 12157 Berlin, Germany

3 Department of Anesthesiology and Intensive Care, Vivantes Klinikum Am Urban, Dieffenbachstraße 1, 10967 Berlin, Germany

4 Department of Infectious Diseases, St. Joseph Krankenhaus, Wüsthoffstraße 15, 12101 Berlin, Germany 